\title{
Assessment of vitamin D status and genetic variation in vitamin-D related genes: key findings from a UK paediatric non-alcoholic fatty liver disease population
}

\author{
P. Gibson ${ }^{1}$, E. Fitzpatrick ${ }^{2}$, A. Quaglia ${ }^{2}$, A. Dhawan ${ }^{2}$, H. Wu ${ }^{1}$, K. Hart ${ }^{1}$, S. Lanham-New ${ }^{1}$ \\ and J. B. Moore ${ }^{1}$ \\ ${ }^{1}$ Department of Nutritional Sciences, Faculty of Health and Medical Sciences, University of Surrey GU2 $7 X H$ \\ and ${ }^{2}$ Paediatric Liver Centre, Kings College Hospital, SE5 9PJ
}

There is growing recognition that vitamin $\mathrm{D}$, in addition to being essential for bone health, also plays key roles in maintaining the balance of human health over disease. Recent research has identified an association between low vitamin D status and non-alcoholic fatty liver disease (NAFLD). Specifically, studies in American ${ }^{(1)}$ and Italian ${ }^{(2)}$ adults with NAFLD have shown low circulating levels of vitamin D associated with the prevalence of NAFLD, which has also been observed in a small cohort of Italian children ${ }^{(3)}$. The aims of this study were to determine, in a UK paediatric population: (i) 25-hydroxvitamin-D [25(OH)D] status; (ii) genotype key polymorphisms related to vitamin D metabolism and NAFLD.

Medical records of 120 NAFLD patients from the King's College Hospital Paediatric Liver Clinic were retrospectively analysed. DNA was isolated from 103 archival blood samples for genotyping polymorphisms in genes (NADSYN1, GC, CYP2R1 and VDR) that influence vitamin D status, in addition to PNPLA3 associated with NAFLD. Biopsies were scored by a liver histopathologist according to the Kleiner/Brunt system ${ }^{(4)}$. Vitamin D seasonality was normalised using the Sachs model ${ }^{(5)}$. 25(OH)D status was assessed by chemiluminescent immunoassay. The study received full NHS ethical approval and was conducted to the Helsinki Declaration.

Cycling of 25(OH)D levels throughout the year was evident; with the majority of samples in the deficient (UK Department of Health; $<25 \mathrm{nmol} / 1,31 \cdot 8 \%$ ) or insufficient (USA Institute of Medicine; $<50 \mathrm{nmol} / 1,84 \cdot 1 \%$ ) ranges. Patients had significantly lower $25(\mathrm{OH}) \mathrm{D}$ levels in winter months when compared to spring, summer and autumn months $(p=0 \cdot 0057 ; p=0 \cdot 0001 ; p=0 \cdot 0001$ respectively). In uni-variate analyses, there was no correlation of $25(\mathrm{OH}) \mathrm{D}$ with ALT, AST, GGT or NAFLD activity score (NAS). In Caucasian patients, the PNPLA3 G allele was associated with increased steatosis and inflammation $(p=0 \cdot 0125$ and $p=0 \cdot 0264$ respectively) whereas the NADSYN1 A allele was associated with increased steatosis $(p=0 \cdot 0451$, Fig. 1a). While no association between the GC rs2282679 and CYP2R1 rs10741657 polymorphisms and NAFLD histological severity was observed (Fig. 1b and Fig. 1c); examination of additional variants of the GC and VDR genes is on-going.

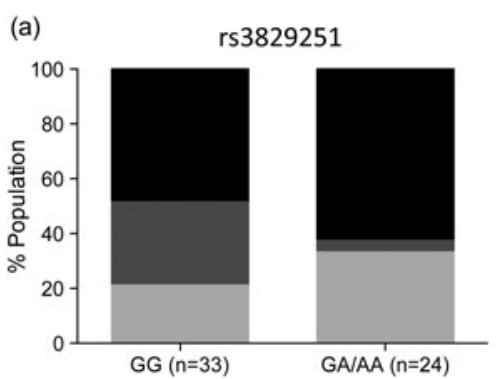

Fig.1a. NADSYN1 Steatosis

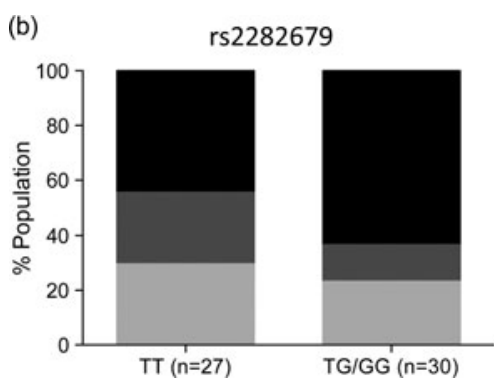

Fig.1b. GC Steatosis

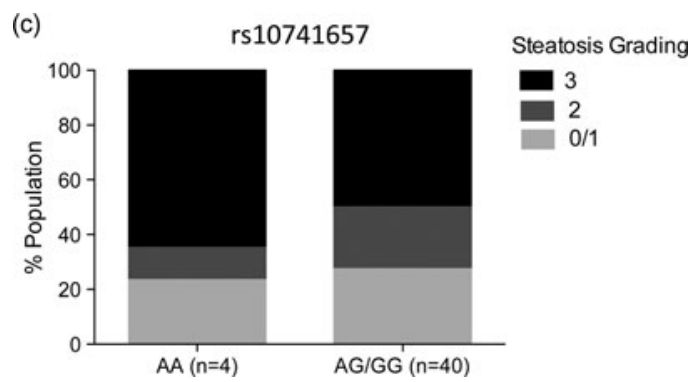

Fig.1c. CYP2R1 Steatosis

In conclusion, UK paediatric NAFLD patients had extremely low winter vitamin D status, and were insufficient throughout the entire year. Interestingly, presence of the NADSYN1 G allele was associated with steatosis severity in Caucasian patients and warrants further investigation.

1. Jablonski et al. (2013) Nutrition, Metabolism and Cardiovascular Disease 8, 792-8.

2. Barchetta et al. (2011) BMC Medicine 9, 85-91.

3. Manco et al. (2010) Hepatology 51, 2229.

4. Kleiner et al. (2005) Hepatology 41 (6), 1313-21.

5. Sachs et al. (2013) American Journal of Clinical Nutrition 97, 1243-51. 